(ICII)-19383

Comparison of the Initial ETA Gas-Propagation Experiments With Theoretical Models

Frank W. Chambers

John C. Clark

Thomas J. Fessenden

Apri1 20, 1982

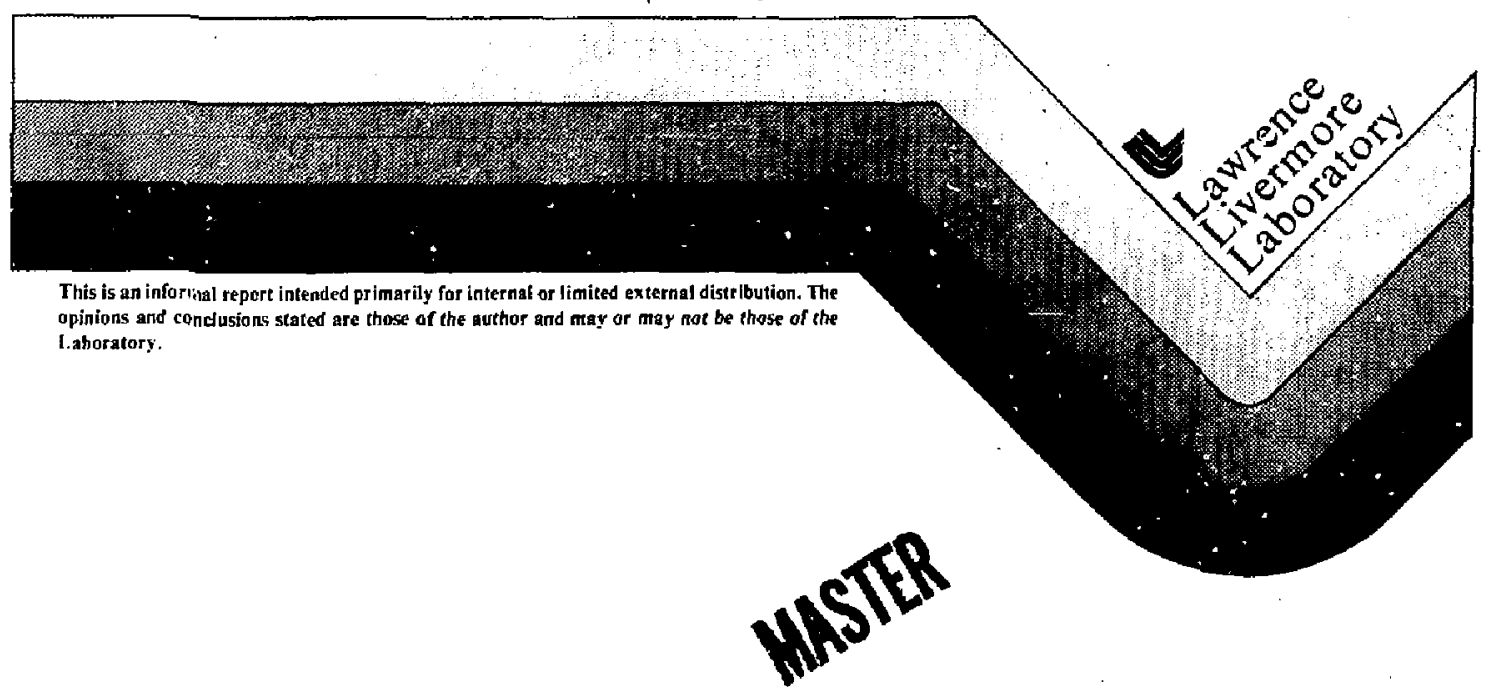

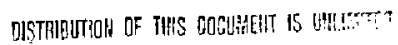


UCID--19383

DE\&2 015415

\title{
COMPARISON OF THE INITIAL ETA GAS PROPAGATION EXPERIMENTS \\ WITH THEORETICAL MODELS
}

\author{
F. W. Chambers, J. C. Clark and T. J. Fessenden \\ Lawrence Livermore Natiana] Laboratory, University of CaTiforria \\ Livermore, California 94550
}

\section{ABSTRACT}

This report contains a description of the initial ETA propagation experiments in air at a beam current of $4.5 \mathrm{kA}$. The beam was abserved to propagate at the pressures anticipated on the basis of previous theory and experiment. A comparison of measured net current waveforms with predictions of the PHOENIX cade showed good ajreement over the pressure range 0.1 to 200 torr. However, the beam was observed to expand with $Z$ at a faster rate than thecry predicts. Excessive transverse beam modulation at injection complicates the experiments and limited their comparison with theory.

* Lawrence Livermore National Laboratoiy is operated by the University of California for the Department of Energy under Contract No. W-7405-Eng-48.

This work is performed by LLNL for the Department of Defense under DAPPA (DOD) ARPA Order 3718, Amendment \#12, monitored by NSWC under Contract No. N60921-80-WR-W0188.

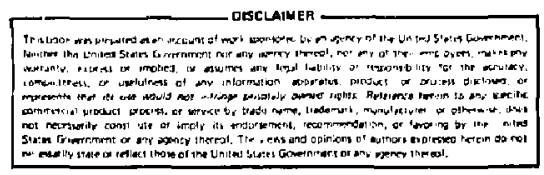


SECTION A. - EXPERIMENT, DIAGNOSTICS, OBSERVATIONS

\section{Introduction}

Initial ETA propagation experiments began in April and continued through May and early June of 1981. At that time the spark gap trigger level had been increased by approximately $40 \%$ with the aid of a new magnetic trigger generator developed primarily by Birx and Reginato. The troublesome Dielectric Science trigger cables had also just been replaced by prototype Beldon cables of the kind intended for use on ATA. We were in the process of adding the beam break-up (BBU) dampening modifications to the ETA accelerator cavities. As a corisequence, the data presented in this report was obtained with two of the eight cavities dampened.

As a result of the above improvements the accelerator performance was considerably improved although, as these experiments demonstrated, the accelerator performance was still not good anough to allow realty meaningful propagation experiments.

As a consequence of the BBU mode the beam had transverse oscillations at frequencies near $830 \mathrm{MHz}$. Perhaps a more significant effect was a transverse sweep generated by slight differences in the firing times of the two Blumleins that drive each coupled pair of accelerator cavities. This timing difference moved from one to more than five nanoseconds and generated large transverse currents in the accelerator cavities that kicked the beam from side to side. Thus, at the point of injection of the beam into gas the initial conditions for the transyerse hose instability were anything but small!

Nonetheless, these experiments did show indications of the propagation windows observed by us and others in the Astron and FX-25 experiments. (1) Their major contribution was to re-emphasize the importance of removing all transverse bean oscillations before injecting the beam into the gas.

Although these experimental results are preliminary, comparisons with theory are useful both to validate (and improve) the theoretical models and to 
highlight the pivotal measurements to be made in future experiments. In the analysis of the data, we considel first the air chemistry modeling including the two-stream instability. Then the propagation phenomena such as matching, erosion, sausage and hose are coripared with our propagation models.

\section{I1. Experimental Description}

For the experiments reported in this report, the experimental cell consisted of several lengths of 6 inch diameter lucite tubing lined with copper screen. Figure 1 shows a photograph of the cell and Fig. 2 shows a schematic layout with dimensions and identification of the primary diagnostic elements. The overall length of the test cell was 4 meters.

The beam entered the cell through a $0.030 \mathrm{~mm}$ thick titanium foil that was located between a pair of beam current monitars labeled \#9 and \#10 in Fig. 2. The first measured the current from the accelerator on the vacuum side of the foil. The second measured the net current on the gas side which is the algebraic sum of the beam current and the return current in the gas. The current monitors \#11 and \#12 measured net current at their respective positions of 1.4 and 4 meters from the entry foil. Between current monitors \#12 and \#13 there was a second foil of $.051 \mathrm{~mm}$ thick Kapton. Current monitor \#13 was in vacuum so it measured the beam current propagating through the 4 meters of gas. .

\section{II. Beam Propagation Diagnostics}

The main diagnostic tools used in these propagation experiments were the television systems and the current monitors.

The TVs viewed the beam generated gas light through the transparent lucite tube and copper screen. Television system TV-1 consists of a microchannel plate intensifier followed by a silicon intensified target (SIT) TV tube. The intensifier can be gated on in a few nanoseconds so that the iv 
system could view a time window variable from 5 nanosecands to several milliseconds. By adjusting the gate timing, this time viewing window can be adjusted to look at any selected portion of the beam pulse. However, in all the experiments reported in this report, the time viewing window was approximately 100 ns. Thus, the data obtained was essentially averaged over each beam pulse. This TV system also has a signal processor that allows a single horizontal and a single vertical scan to be displayed on a dual trace oscilloscope. This scan processor was used to measure the size of the propagating beam within the gas. The position of these scans can be varied anywhere within the TV field of view. This TV also has a zoom lens that could be remotely varied over a 10:1 focal length from $15 \mathrm{~mm}$ to $150 \mathrm{~mm}$. Television system TV-2 was a standard CCTV that had no gating capability.

The current monitors measure the return current in the cell wall by measuring the voltage drop across a .0013 $\mathrm{mm}$ thick stainless steel foil that forms a $3 \mathrm{~cm}$ section of the cell wall. These monitors are an LLNL design(2) and are individualiy calibrated with a nominal response of $1 \mathrm{~V} / \mathrm{kA}$. Their risetime response is less than 1 nanosecond. In addition to measuring the net current in the pipe, they can also be used to measure the position of the beam centroid.

In performing the gas propagation experiments the cell was filled to the desired pressure from a compressed gas bottle through an externally controlled leak valve. The desired pressure was monitored with a Baratron pressure gauge that can measure pressure in the range from $1 \mathrm{~m}$ torr to $1000 \mathrm{torr}$. The actua] experimental range was fram $30 \mathrm{~m}$ torr (the minimum pressure obtainable with the lucite tubing) to 600 torr.

\section{Initial Beam Conditians}

The beam current measured with current monitor \#g at the entrance of the experimental cell showed a peak current of $4.5 \mathrm{kA}$ with a FWHM of 25 nanoseconds. The risetime of the pulse measured $2 \mathrm{~ns}$ from .5 to $3 \mathrm{kA}$. There was a 5 ns "front porch" of about 300 amps. 
These measurements were taken during the time that the ETA was being modified to reduce the BBU instability. Only two cavities of the 8 accelerator units had been modified at the time. Therefore, the current in the accelerator was reduced to where the $\mathrm{rf}$ fluctuations of the bean at $830 \mathrm{MHz}$ were not significant. There was still a problem of beam sweep in which the beam was deflecting approximately $.8 \mathrm{~cm}$ at the entry foil during the beam pulse.

The beam energy at the accelerator output was $3.8 \mathrm{MeV}$. The emittance measured at the end of the accelerator gave a $q$ of $70 \mathrm{mr}-\mathrm{cm}$. The Ti entry foil adds additional scattering that must be included for predicting propagation phenomena. The mean square angle, $\left\langle\theta^{2}\right\rangle$, is about $5.4 \times 10^{-3}$. The radius of the beam at the foil can be estimated to be $1.5 \mathrm{~cm}$ by extrapolating back from data at $20 \mathrm{~cm}$ distance. Combining this with the emittance measurement from the accelerator gives a $q$ at injection into the gas cell of $112 \mathrm{mr}-\mathrm{cm}$.

The gas pressure in the tank was varied from .03 to 200 torr in a $1,2,5$ sequence during which the bean current and position at each of the beam monitors were recorded an an oscilloscope. Also a picture of the beam in gas was obtajned with the aid of TV-1.

\section{Experimental Results}

The peak current recorded at each current monitor was plotted as a function of pressure and is shown in Fig. 3. The input cirrent at current monitor \#9 was constant at $4.5 \mathrm{kA}$ and is shown as a dotted line in the top plot. The current at monitor $\$ 10$ is shown in the top plot. At pressures below 0.2 torr, current neutralization is jess than $30 \%$ and rather abruptly increases to $70 \%$ between 0.2 to 0.5 tory with a maximum at about 1 torr. As pressure increased the fraction of current neutralization reduced to zero at 200 torr. This resuli is essentially the same as observed in earlfer propagation experiments on the $F X-25(1)$. The current at monitor $\$ 11$ at 1.42 meters into the gas shows a region of current multiplication where the net. 
current was measured to be as much as two times the beam current. This was identified as being due to a two-stream instability in previous experiments with the ASTRON accelerator and with the $F X-25(1)$.

Monitor \#13 measured only the beam currerit since it is in vacuum. At low pressure where the two-stream instability is strong the current measured may include some energetic plasma electrons that gained enough energy to pass through the back foil. Previous experiments on the FX-25 under two-stream conditions showed that plasma electrons gained energy to greater than $50 \mathrm{keV}$. No effort was made to confirm that phenomenon in these experiments.

The significant point of the current measured at monitor \#13 is that there are two pressure regions where the bearn propagated significant ly we 71 over the entire 4 meters of gas. The region from .03 to .2 torr has been termed the Ion Focus Regime (IFR) in which the secundary electrons directiy produced by beam electrons are rapidly expelled from the beat, channel by the strong radial electric fields at the beam head. The remaining ions allow the beam to pinch to a stable propagating mode. The second region of good propagation was centered at af proximately 1 torr in these experiments. This propagation window is similar to that observed with the FX-25 beam (1). However, the optimum pressure observed with the $F X-25$ beam was centered at 2 torr in air.

The TV-I pictures of beam propagation are shown in Fig. 4. For pressures below 0.5 torr there was not enough light emitted from the gas to observe the beam. The TV zoom lens was adjusted to show about one meter of propagation for these pictures, although all views are not the same. The spot in the center of the pictures is the view of the optical port located 0.72 meters from the entry foil as shown in fig. 2.

With the optimum propagation pressure at 1 torr the beam travels a straight path. Above 1 torr the beam begins to deflect more grossly as the pressure is increased. At 10 torr and above, the beam appears to be spreading out very rapidly as it propagates down the tank. This is 1ikely due to an 
integrated light effect of the beam hosing up and down. The hosing of the bean was observable by the beam monitors $\# 11$ and $\# 12$. As shown in Fig. 4, at pressures above 10 torr both the TV light intensity and the beam size increase rapidly with distance from the entrance foil.

The scan line feature of the TV was used to measure the beam dianeter at selected positions along the tube. Figure 5 shows the measured beam radius from $20 \mathrm{~cm}$ to $140 \mathrm{~cm}$ for the beam in 2 torr air. The scan line feature was a7so used to measure the radius of the beam at $60 \mathrm{~cm}$ from the foil as a function of pressure. This is shown in Fig. 8 where the radius is compared with theoretical predictions.

\section{SECTION B. - COMPARISON OF THEORY AND EXPERIMENT}

\section{Air Chemistry - Description of Theory}

Air chemistry models usually assume local evolution in the lab frame. In this case, the field behavior is completely determined by the beam current density, $J_{B}(r, x)$. Here $r$ is the radial coordinate, $x$ is the distance back from the beam head. The models allow one to predict $n_{e}(r, x), \sigma(r, x)$, $\leftrightarrow(r, x), J_{p}(r, x) B_{\theta}(r, x)$ etc. where $n_{e}$ is the plasma electron density, o the conductivity, $A$ the usual pinch potential $(3), J_{p}$ the plasma current density, and $B_{\theta}$ the pinch magnetic field.

Theoretical calculations of the field quantities are done using the PHOENIX fieldsolver code ${ }^{(4)}$. The parameters for $J_{B}(r, x)$ used to model the ETA beam are specified in Table I. Since measurements were made near the injection point, the current parameters for ETA are for a cylindrical beam with no head. The rise and fall lengths are determined approximately from the $I_{B}(X)$ trace in vacuum. The "front porch" observed on $I_{B}(X)$ has not been included. The PHOENIX code computes $l_{N E T}(X)$ for comparison with the observed current monitor output. 


\section{Air Chemistry - Theory vs Experiment}

Computed results are coripared with experiments in Fig: 6 and Fig. 7 . Figure 6 compares the maximim net current, INAX, (maximized in $X$ ) versus pressure, In Fig. 7 the $X$ avolution of the experimentally observed net current is compared with the theoretical prediction at each pressure from $.1 \mathrm{~T}$ to $200 \mathrm{~T}$.

Good agreement between theory iriv experiment is obtained in Fig. 6. Theoretical results show a greater dependency on pressure than does the experiment but the trends are in good agreement. The falling net current as pressure is reduced from 100 torr to 1.0 torr is due to increased conductivity generation by avalanche ionization and an increase in the maximum conductivity ceiling due to recombination. Below 1 torr the rate of avalanching is reduced as starvation occurs. The code predicts a rise in net current. However, models and assumptions employed in the conductivity calculations are known to fail at these pressures. The agreement at .2 torr is fortuitous as will be seen in the $X$ histories in Fig. 7 .

Figure 7 displays the $X$ development of $I_{\text {NET }}$ as measured and calculated. The calculations give $\left(I_{N E T} / 4.5 \mathrm{kA}\right)$ versus $X$ in centimeters to $1000 \mathrm{~cm}=33 \mathrm{~ns}$. The scope traces are all $1 \mathrm{kA} /$ division vertically. The horizontal sweep rate is varied between $5 \mathrm{~ns} /$ div. $(150 \mathrm{~cm} /$ div) and $20 \mathrm{~ns} /$ div $(600 \mathrm{~cm} /$ div). The location of the trigger varies from pressure to pressure. In several cases the multiple traces indicate variations in the beam from shot to shot. The actual accelerator pulse contains a "front porch" which has not been included in the idealized $\mathrm{J}_{\mathrm{B}}(x)$. The scatter and uncertainty in the data is sufficient that digitizing and replatting it is not justified at this time.

The observed current, $I_{N E T}(X)$, can be roughly described by its rate of rise in $X$, maximum value attained, and decay rate after the beam pulse ends. At low pressures the interplay of avalanche ionization and drect deposition determine the rate of $r$ ise. Saturation in o due to recombination will occur at varying $x$ values for different radial positions. The maximum current reached, I IAXT, is determined by the interplay of all three processes. After 
the beam current turns off the $I_{\text {NET }}$ decay is determined primarity by the magnetic decay length:4), $\lambda_{m}=4 \pi \sigma a{ }^{2} / c$. The decay length is a measure of maximum conductivity produced and thus of the recombination rate.

Qualitative agreement is good between theory and experiment down to about 1 torr in pressure. The decay length for $I_{N E T}$ after the beam turns off at $X=600 \mathrm{~cm}$ is seen to increase with decreasing pressure in both theory and experiment. Decay rates appear very comparable. Further, the decay rates agree with the $\lambda_{m}(X=600)$ determined in Table 2 from the computed on-axis conductivity at $X=600 \mathrm{~cm}$. In this tail region the current is decaying on a $\lambda_{\mathrm{m}}$ distance scale(4). Simultaneously, the conductivity $\sigma$ is decaying due to recombination. This decay is parameterized by $\lambda_{\sigma}=1.0 /\left(\frac{1}{\sigma} \frac{\Delta g}{\Delta x}\right)$, although the decay is algebraic and not exponential. Since at all pressures $\lambda_{m} \ll \lambda_{\sigma}$ one is justified in assuming constant $\sigma$ during the decay of $I_{N E T}$. For the runs with $p \geq 5$ torr the on-axis conductivity is nearly constant after $X=100 \mathrm{cmi}$ due to recombination. The apparent agreement in both the rise in $I_{\text {NET }}$ for $100<X<600 \mathrm{~cm}$ and the fall after $X>600$ indicates the recombination rate is fairly well described by the model.

At 20 torr and below the theoreticai predictions show a rise and fall in $I_{\text {NET }}$ during the first $50 \mathrm{~cm}$ in $x$. The fall occurs when rapid avalanche ionization causes a sharp increase in $\sigma$. The $E_{Z}$ eiectric field does not fall as fast so the return current increases and the net current falls, Code runs where "porch" has been added to $I_{B}(X)$ do not display this initial spike. Other theoretical current diagnostics such as $I_{E F F}$ do not display this initial jump. This phenomena is not observed in the experimental data, and is a consequence of the sharp current rise in the model.

Code predictions look accurate down to about 1 torr. At this pressure most approximations made in modeling plasma and conductivity behavior are failing(6). One must be cautious, however, agieement on the macroscopic

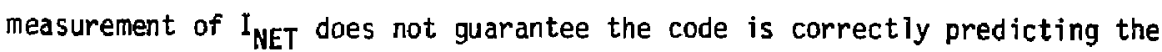
microstructure in $(r, x)$ of the fields. Details of this microstructure may affect propagation (5). 


\section{Air Chemistry Summary}

In sumary, ETA experiments are very useful for calibrating air chemistry models. Calculations using present beam, direct deposition, avalanche ionization, and recombination models are in good agreement with the experiment. At this point, for pressures above 5 torr, the models appear to need only "fine tuning". Neither the present data nor the limited analysis is sufficient to perform the fine tune. This work will be a lang and sometimes tedious task which should be done when manpower and data are available.

For future experiments several improvements would be useful. Data is needed on a fast time scale to study beam front conductivity generation which is critical to several propagation questions. A clean measure of $I_{\text {NET }}$ during the bean pulse is needed to determine $\lambda_{\mathrm{m}}(X)$ and hence the saturated conductivity. Finally, a measurement of the decay of $I_{\text {NET }}$ to fairly long times $(\sim 30-100 n s)$ is required to measure $\lambda_{m}\left(x>L_{p}\right)$ and to observe how recombination affects $\sigma$ and $I_{\mathrm{NET}}$. These measurements should again be made near the entrance foil $\left(Z<\lambda_{B}\right.$ where $\left.\lambda_{B} \sim 20 \mathrm{~cm}\right)$ to reduce propagation effects. One cannot approach the foil too closely or the field equation models fail. Corollary measurements should he made as close as possible to the $Z$ at which $I_{\text {NET }}$ is measured. Fringing magnetic fields from the final focusing coil are not important since one is not concerned with beam propagation and at these pressures the plasma is collision dominated.

Further diagnostics such as $\int n_{e} d l$, the integrated line density, would be useful far comparison with codes. Of particular use would be the radial and temparal resolutio: of beam and plasma currents; $J_{B}(r, x), J_{p}(r, x)$. This could allow the local comparison of theory and experiment. The local quantities are more sensitive to the air chemistry models than is $I_{N E T}$. It is now appreciated $(5)$ that the location is as important as the magnitude of the net current in determining beam propagation characteristics. 


\section{Two Stream}

The two stream mode for a beam in air is collisionally stabilized at sufficientiy high pressures (7). At sufficiently low pressures the beam will not produce $f(1) 1$ charge neutralization and there can be no two stream instability. Using a simple dispersion relation we find two stream should be present between .01 and 1.2 torr.

These stability boundaries agree well with the observed boundaries at about .03 torr and 1 torr. The two stream analysis involves many caveats and approximations - the agreement is therefore somewhat fortuitaus. The proximity of the low pressure window to the unstable region suggests there may be some vestiges of the instability generating conductivity and facilitating propagation.

There is no realistic, complete description of the $x, z$ evolution (linear or otherwise) of the two-stream instability in the inhomogeneous beam. The instability is convective since the amplitude of the current multiplication is larger at the downstream beam bugs. The increased net current observed is in accord with the two-stream oriven current multiplication theory ${ }^{(8)}$.

In future experiments the study of the two-strean is not a priority item. The key unresolved theoretical question is the scaling of the pressure boundary with $\gamma$. This will require ATA and larger accelerators. Two-stream studies can be performed at other facilities but one does require a pinched, propagating beam.

\section{Propagation}

Propagation phenomena to be examined are the developement of an equilibrium, the slow evolution due to Nordsiecking and erosion, and the stability of the equilibrium to sausage and hose modes. Due both to the low beam energy and the paucity of data only brief qualitative comparisons will be made. 
VI. Matching to an Equilibrium

Using a simple energy conservation model one can predict the equilibrium radius versus net current (9). An even simpler approach using conservation of emittance is adequate at present. Assuming the beam equilibrium radius with no return current to be $a_{0}$ one finds

$$
a^{2}=a_{0}^{2}\left(\frac{I_{B O}}{I_{\text {NET }}}\right) \text {, }
$$

Here $a_{0}=q_{0} /\left(I_{B O} / I_{A l f v e n}\right)$ and $q_{0}$ is the quality of the beam injected into the gas.

This prediction for a is plotted in fig. 8. This number corresponds to an Emititance $q_{0}=115 \mathrm{mr}-\mathrm{cm}$.

The reasonably good agreement between observed and predicted beam radii indicates the beam is in a self-pinch equilibrium. This equilibrium is achieved within several betatron wavelengths of the jnjection point.

The measurement of beam radius is at $Z=60 \mathrm{~cm}$ while the net current measurements were made at $Z=0 \mathrm{~cm}$ and $Z=140 \mathrm{~cm}$. At $140 \mathrm{~cm}$ the net current at .20 torr is $7 \mathrm{kA}$ due to two-stream current multiplication $(8)$. If this large net current is present at $Z=60 \mathrm{~cm}$ it could account for the minimum in radius observed at .2 torr. To facilitate understanding of results one should make measurements of the observables as close together in $Z$ and $X$ as is possible.

\section{Evolution of the Equilibrium Radius}

The evolution of beam radius away from the foil shown in Fig. 5 can not be explained by the usual processes; energy loss and Nordisecking. The Nordisecking length for a beam is:

$$
L_{N}=2.07 \mathrm{~m}(y / 10)\left(I_{N E T} / 10 \mathrm{kA}\right)(760 \text { torr } / \mathrm{p})
$$


which evaluates at 2 torr with $\gamma=8.6$ and $I_{\text {NET }}=1.7 \mathrm{kA}$ to $L_{N}=1.1510^{4}$ $\mathrm{cm}$. Energy loss can also cause the beam to expand with an effective expansion length

$$
L_{E}=2 \gamma /(d \gamma / d Z)
$$

From PHOENIX runs one finds $E_{Z}$ near the beam tail to be $\sim 1$ stat volt/cm so $\mathrm{d} \gamma / \mathrm{dZ}=\mathrm{eE}_{\mathrm{Z}} / \mathrm{mc}^{2}=5.8510^{-4}$ and $\mathrm{L}_{\mathrm{E}} \simeq 2.910^{4} \mathrm{~cm}$. Neither classical process can explain the observed expansion.

Several speculative explanations can be suggested. Perhaps the diagnostic is in error, this cauld be checked with the pirhale Faraday cup. The beam may have been injected with a positive expansion velocity and not at a neck. The good correlation between $I_{N E T}$ and a in Fig. 8 makes this explanation unlikely. Perhaps the diagnostic is observing the unpinched eroded beam front which may be expanding this rapidly. One estimates only 10-80 cm of the beam is unpinched by $140 \mathrm{~cm}$ propagation so this explanation seems unlikely. Another explanation is the presence of an instability which alters but does not disrupt propagation. The apparent beam width may be due to hosing or some vestigal beam sweep from the accelerator. The radial behavior is similar to that observed for sausaging beams at higher currents and pressures. This experiment is one which merits muci closer examination. A measurement of $J_{B}(r)$ with as much $X$ resolution as possible (although $-5 \mathrm{~ns}$ would suffice) is highly desirable. In light of present theoretical understanding, this evolution of beam radius during propagation is the most baffling feature of the current experiment.

\section{Erosion}

The erosion rate for this low energy beam is primarily energy loss driven, the rate being(10)

$$
\frac{d X}{d 2}=.15\left(\frac{I_{N E T}}{I_{B}}\right)
$$


After $4 \mathrm{~m}$ of propagation at 2 torr where $I_{\text {NET }}=1.7 \mathrm{kA}$ one predicts $23 \mathrm{~cm}$ of the beam will have eroded. Some portion of the beam $r$ ise will have blown off also. Thus to measure erosion will require sub-nanosecond resolution of arrival times. Hence, no comparison of theory and experiment is possible at present.

Coupled with the theoretical prediction of erosion rates is the prediction of a "steady state" fully developed nose profile. Experimental confirmation of this profile is highly desirable. One would like to measure for example, $J_{B}(r=0, X), R(X)$ or $E_{Z}(X)$ with sub-nanosecond resolution for a beam which has propagated many betatron wavelengths. Knowledge of the erosion rate is useful for determining range limitations; knowledge of the beam front profile is more essential since stability depends critically on the parameters in the beam nose.

\section{Sausage/Hollowing}

Simulations have shown (5) that high currents (10 kA or greater), short rise times $(-15 \mathrm{~cm}=.5 \mathrm{~ns})$ and intermediate pressures ( -100 torr) were required for the sausage/hollowing instability to occur in a uniform gas. At 2 torr the ETA beam is prejicted to be sausage stable. There is no evidence of sausaging in the experiments. Some preliminary experiments have suggested a possible hollow beam. However, this observation occurred within one betatror wavelength of the foil. The measurement was of the injection condition and not of a beam propagation phenomena.

\section{$x$. Hose}

The hose instability is usually cited as the key propagation question for the lead pulse. This first ETA experiment has given little direct information on the hose instability. A stable propagation window is observed, similar to FX-25 results. Much above 1 torr the beam does not propagate. At one torr 
code runs (EMPULSE, RINGBEARER II) are of questionable value due to air chemistry uncertainty. Theoretical efforts at this time are better spent analyzing the previously discussed air chemistry results.

As gas propagation experiments are repeated and improved several key questions should be studied. Theoretical predictions show the hose mode is always present but it is convective. Experiments to verify (or deny) the convective nature of the low amplitude instability would be of great value. Such an experiment will require a beam which propagates stably for 10 betatron wavelengths. Measurements of instability amplitude versus $x$ with $\approx 1$ nS resolution are needed. Realistical?y, one will probably not sbserve true convection since the hose keeps being excited in the beam head. The evolution to an amplitude which grows in $X$ from pulse head to tail but which does not get progressively worse in $Z$ would suffice to verify the convective nature of the instability.

\section{$X I I$. Conclusions}

The original gas propagation experiments on ETA are useful for verifying severat models and aspects of the theoretical work. This comparison with theory indicates several areas where further experimentation and improved diagnostics will be of greatest utility.

The evolution of beam radius with $Z$ for the $Z$ torr propagating beam cannot be explained theoretically. Also, the strongly increasing light intensity and radius with $Z$ at 10 torr and above suggests that the beam is interacting more strongly with the background gas than wculd be expected from classical processes. Otherwise, the experimental results have verified the theoretical models for the glabal air chemistry at 5 torr and above. Further analys is of this or subsequent data would be of value to further pin down air chemistry models as indicated in the text. A massive effort at data reduction and code sensitivity scans is not justified with the presently available data. 
Future comparison of ETA experiments and theory should proceed with a11 deliberate speed. Emphasis should be on code calibration with respect to air chemistry and development of qualitative understanding of the stability boundaries and space, time evolution of instabilities. Proper interaction of theory and the ETA experiment can greatly raise the confidence level in propagation code predictions as we await the more definitive propagation experiments to be done with ATA. 
Acknowledgement

These data were gathered with considerable assistance from the other experimental physicists and technicians who comprise the ETA experimental team.

We particularly thank Walt Atchison for assistance in measuring emittance and beam energy and Ross Spoerlein, Doyle Rogers and Dave Trimble in tuning and operating the ETA accelerator. 


\section{REFERENCES}

1. R. J. Briggs, J. C. Clark, T. J. Fessenden, R. E. Hester, and E. J. Lauer, in Proceedings of the 2nd International Topicai Conference or, High Power Electron and Ion Beam Research and Technology (Cornell University, Ithaca, N. Y., 1977), Vol. I, p. 319.

2. These monitors have evolved from similar monitors used on the Astron acceleratur and described by Fessenden, Stallard, and Berg, in RSI $\underline{43}$, 1789 , (1972).

3. E. P. Lee, The New Field Equations, UCIC-17286, Lawrence Livermore Nationai Laboratory, October 4, 1976.

4. F. W. Chambers and D. M. Cox, Standard Test Case Runs For The Empulse Mnnopole Fieldsolver and Conductivity Generation Mode1, UCID-19213, Lawrence Livermore Nationa) Laboratory, March 2, 1981.

5. F. W. Chambers, Sausage Mode Stability Boundaries - Enumeration and Verification, UCID-18879, Lawrence Livermore National Laboratory, December 2, 1980.

6. S. S, Yu - private communications.

7. E. P. Lee, F. H. Chambers, L. L. Lodestro, and S. S. Yu, in Proceedings of the 2nd International Topical Conference on High Power Electron and Ion Beam Research and Technology (Cornell University, Ithaca, New York, 1977), Vol. I, p. 381 .

8. F. W. Chambers, Phys. Fluids, Vol. 22, No. 3, p. 483, March 1979.

9. E. P. Lee, Determination of the Radius of a Self-Pinched Beam from its Energy Integral, UC ID-18495, Lawrence Livermore National Laboratory, January 2, 1980.

10. E. P. Lee, Mode1 of Beam Head Erosion, UCID-18768, Lawrence Livermore National Laboratory, August 8, 1980. 
TABLE I

Definitions for parameters:

$I_{B O}=$ maximum bean current

$L_{R} \quad=$ beam rise length

$L_{F} \quad=$ beam fall length

$X_{F} \quad=$ location of beam falloff

$R_{M I N}=$ minimum beam radius as $X \rightarrow \infty$

$R_{\text {MAX }}=$ maximum beam radius as $X \rightarrow-\infty$

$x_{0}=$ location of beam neck

$x_{W} \quad=$ width of beam neck
Values for ETA:

$=4.5 \mathrm{ka}-$ BENNETT

$=30.0 \mathrm{~cm}=1.0 \mathrm{~ns}$

$=60.0 \mathrm{~cm}=2.0 \mathrm{~ns}$

$=600 . \mathrm{cm}=20 . \mathrm{ns}$

$=1.0 \mathrm{~cm}$

$=1.0 \mathrm{cri}$

$=15.0 \mathrm{~cm}$

$=15.0 \mathrm{~cm}$ 
TABLE II

$=0$

200

100

so $4.010^{11}$

esu $\quad 105 \mathrm{~cm} \quad 1.010^{11}$ esu $168 \quad 2.010^{11}$

$3.110^{11}$ esu $\quad 467 \mathrm{~cm}$

$20 \quad 7.5 \quad 10^{11}$

314

$4.610^{11}$

$4.410^{11}$

600

$10 \quad-1.2 \quad 10^{12}$

503

$8.010^{11}$

$7.010^{11}$

834

$\begin{array}{lll}5 & 2.2 \quad 10^{12}\end{array}$

921

$1.410^{12}$

$9.910^{11}$

$1.410^{12}$

1000

$25.0 \quad 10^{12}$

2100

$3.0 \quad 10^{12}$

3140

$5.510^{12}$

$2.210^{12}$

900

$17.510^{12}$

4200

$9.010^{12}$

.5 1. $10^{13}$

4200

$1.810^{13}$

$3.110^{12}$

800

$4.410^{12}$

1300

$7.010^{12}$

3800

.2 1. $10^{13}$

.1 


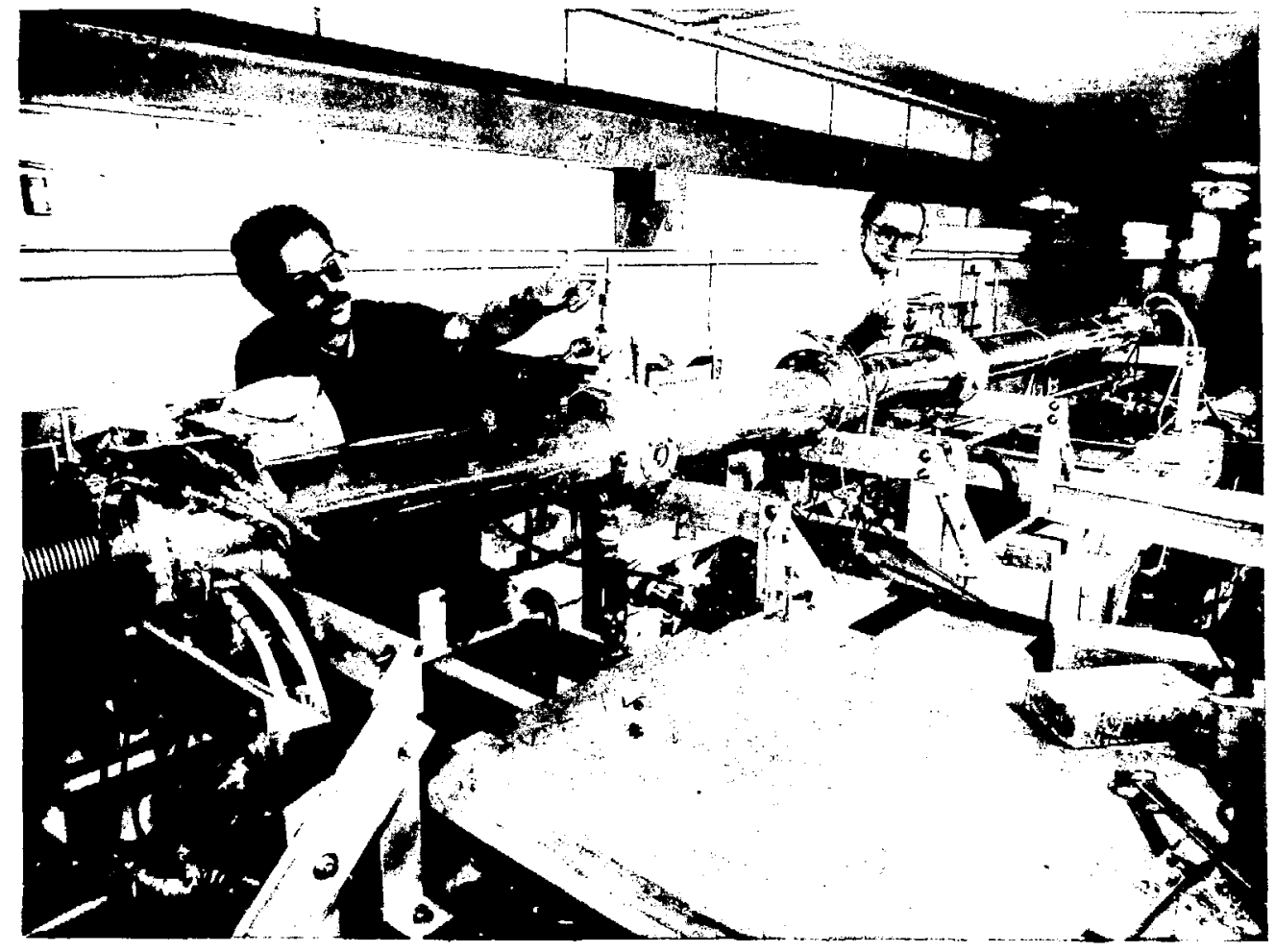

Figure 1. 


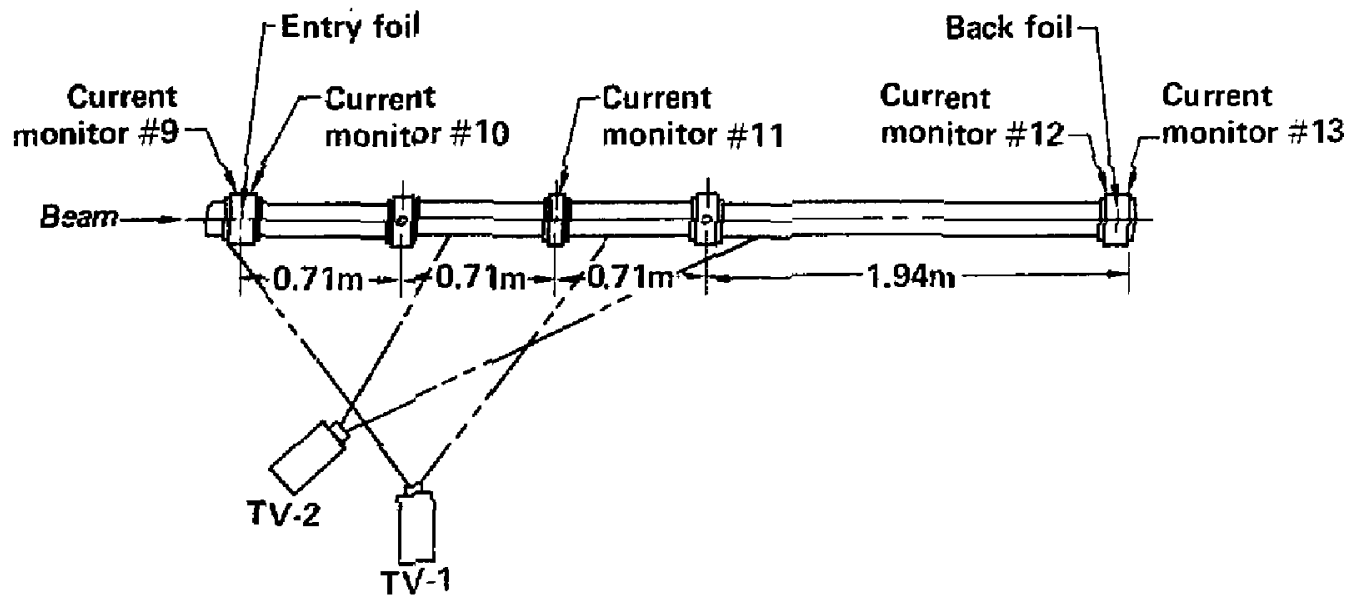

Figure 2. Beam propagation experimental tank. 

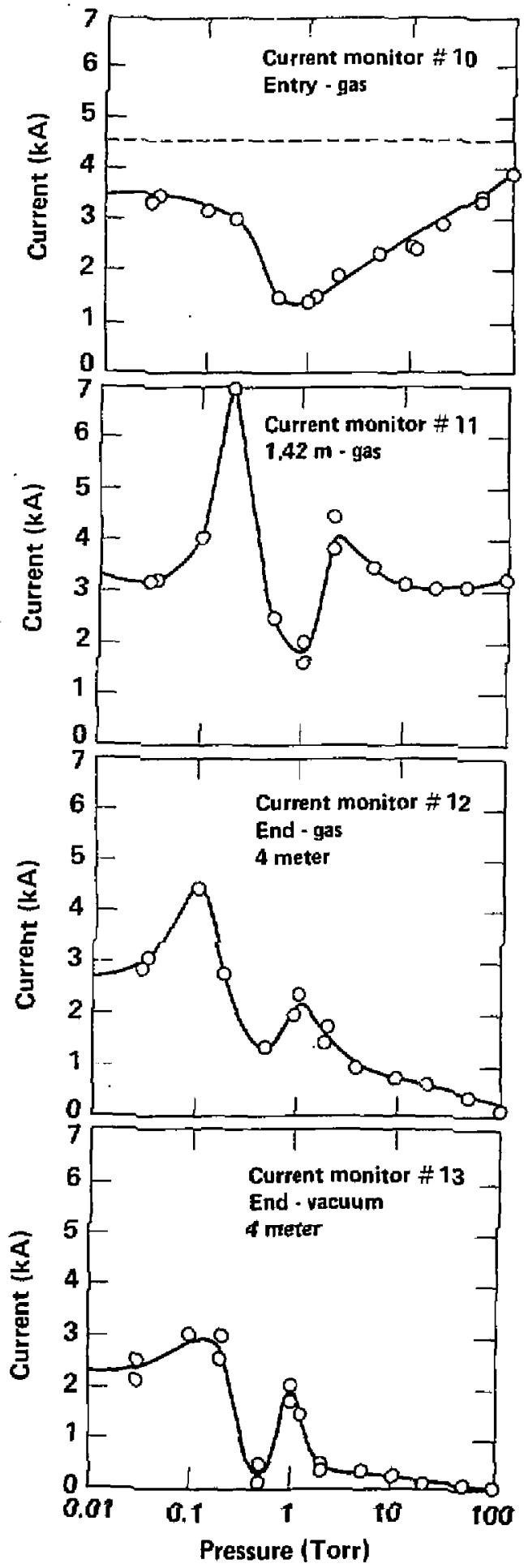

Figure 3. Net current for a $4.5 \mathrm{kA}$ beam propagating in the air ws prescure at different locations in the experimental tank. 


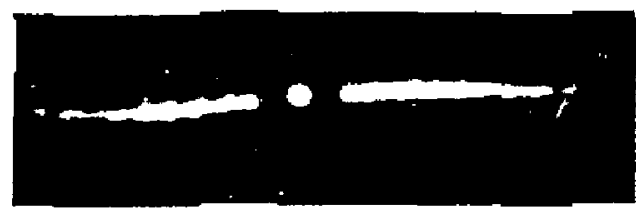

0.5 torr

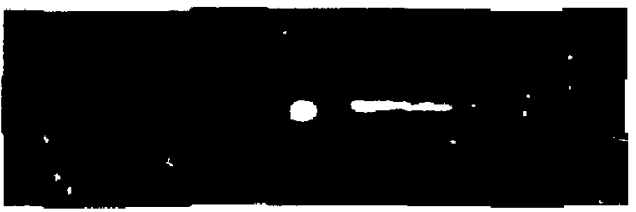

1 torr

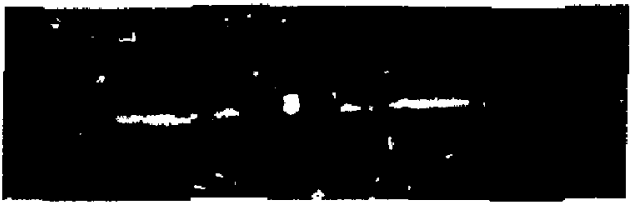

2 torr

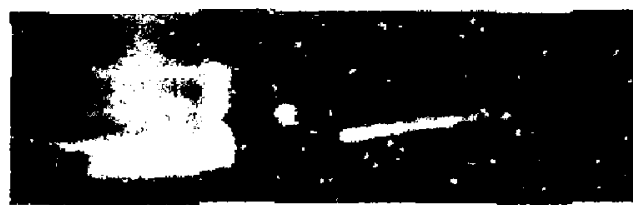

5 torr

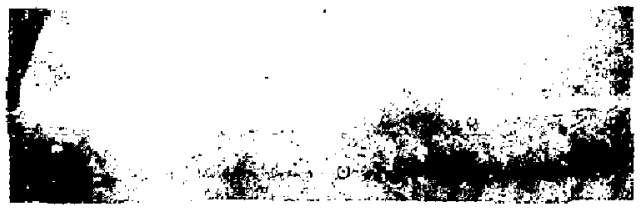

Geam direction

10 torr

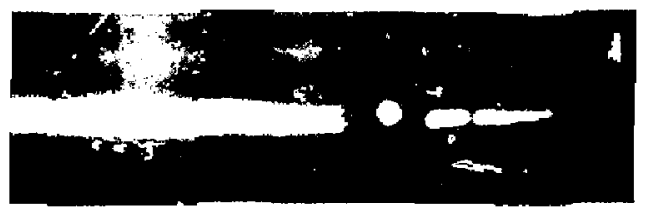

20 torr

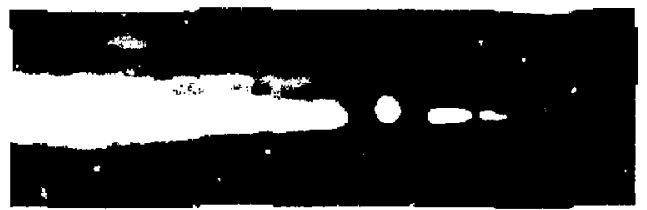

50 torr

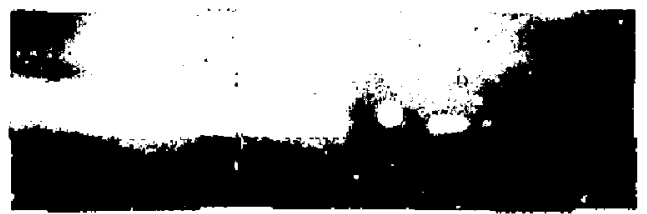

100 torr

Figure 4. 


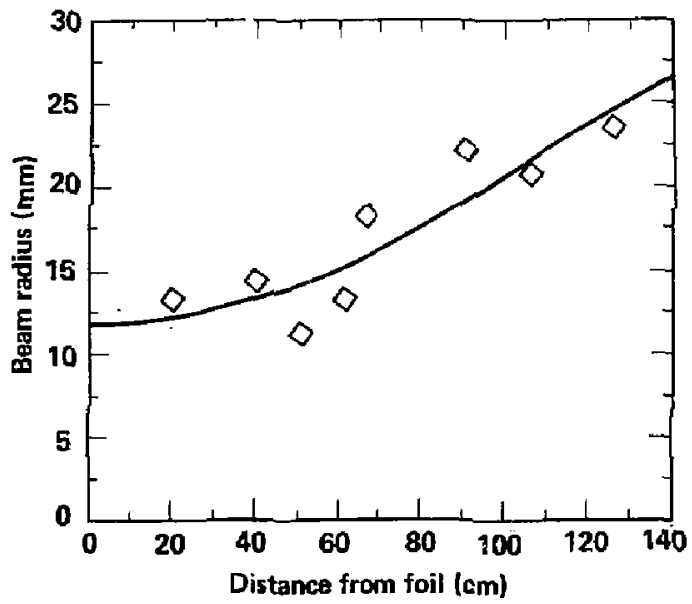

Figure 5. Optically determined beam radius vs distance in 2 torr air. 


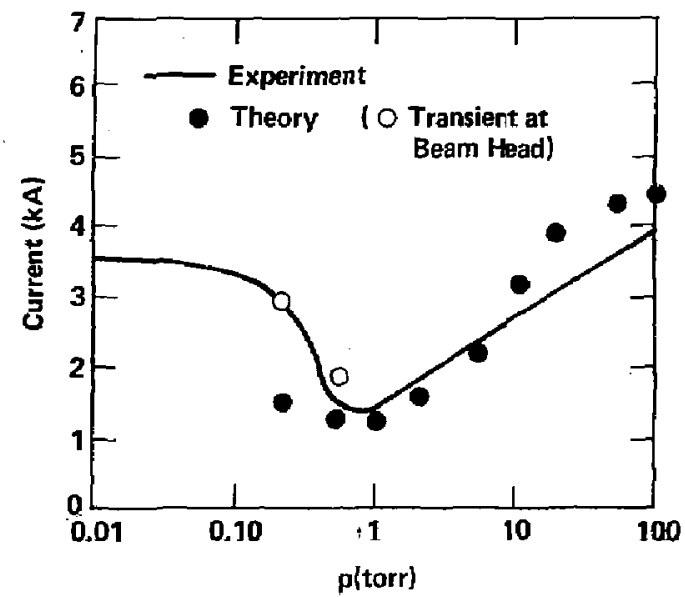

Figure 6. Peak net current vs pressure: theory end experiment. 


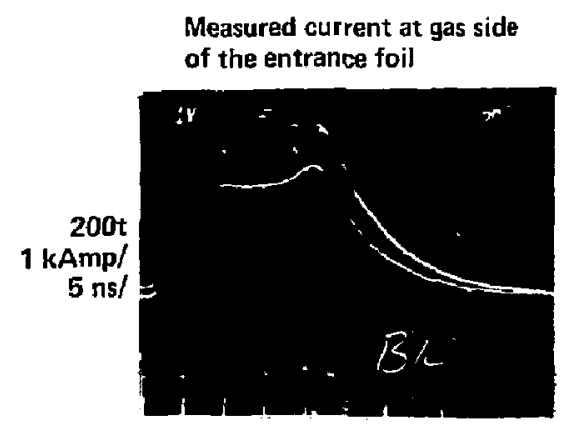

\section{Computed current}
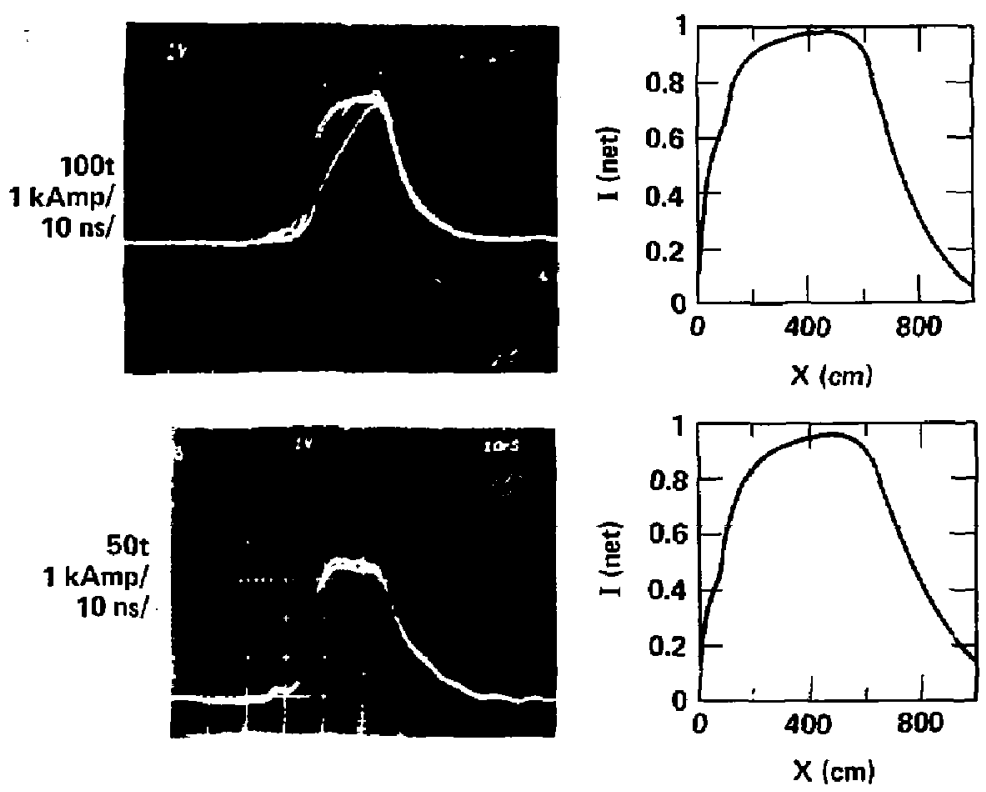

Figure $7 A$. Comparison of theory with experiment at the point the beam enters the gas. 
Measured current at gas side of the entrance foil
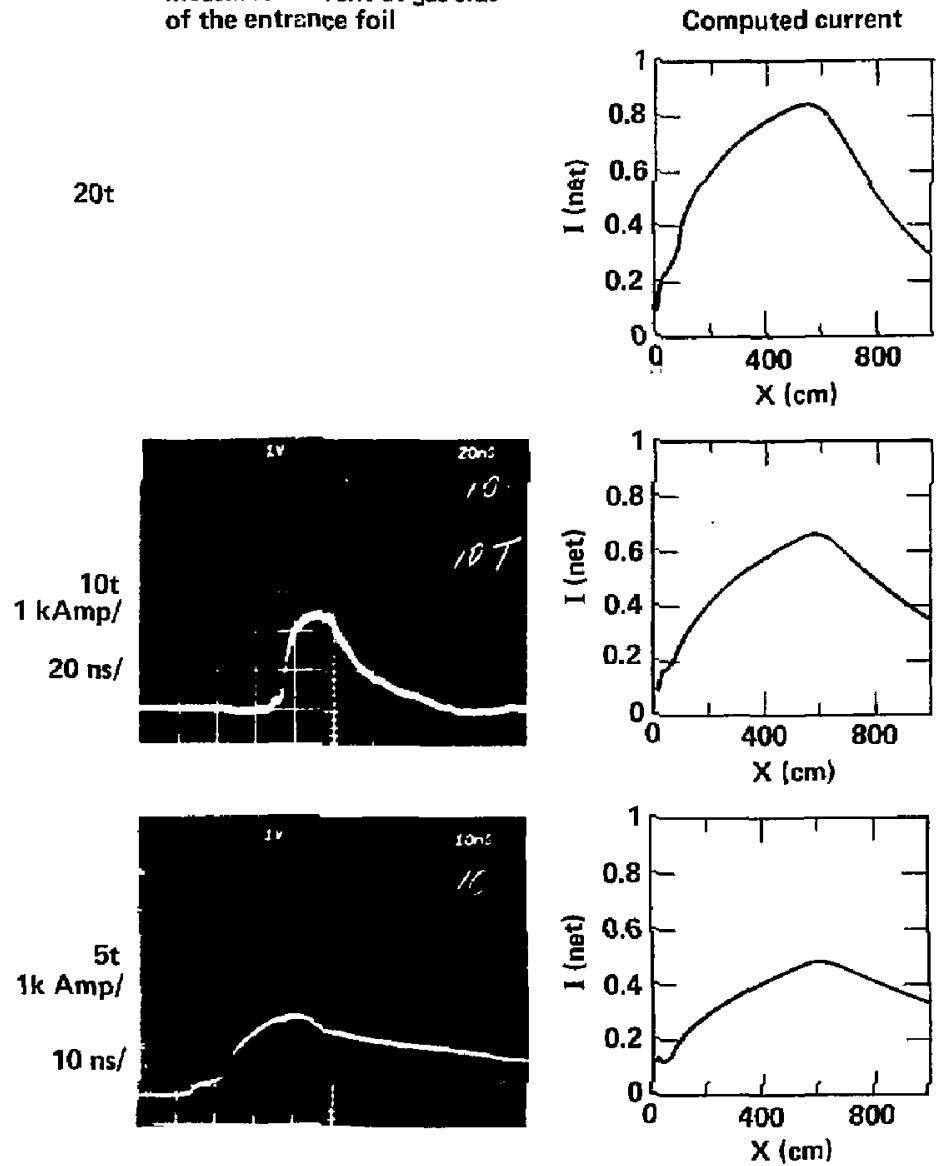

Figure 7B. Comparison of theory with experiment at the point the beam enters the gas. 

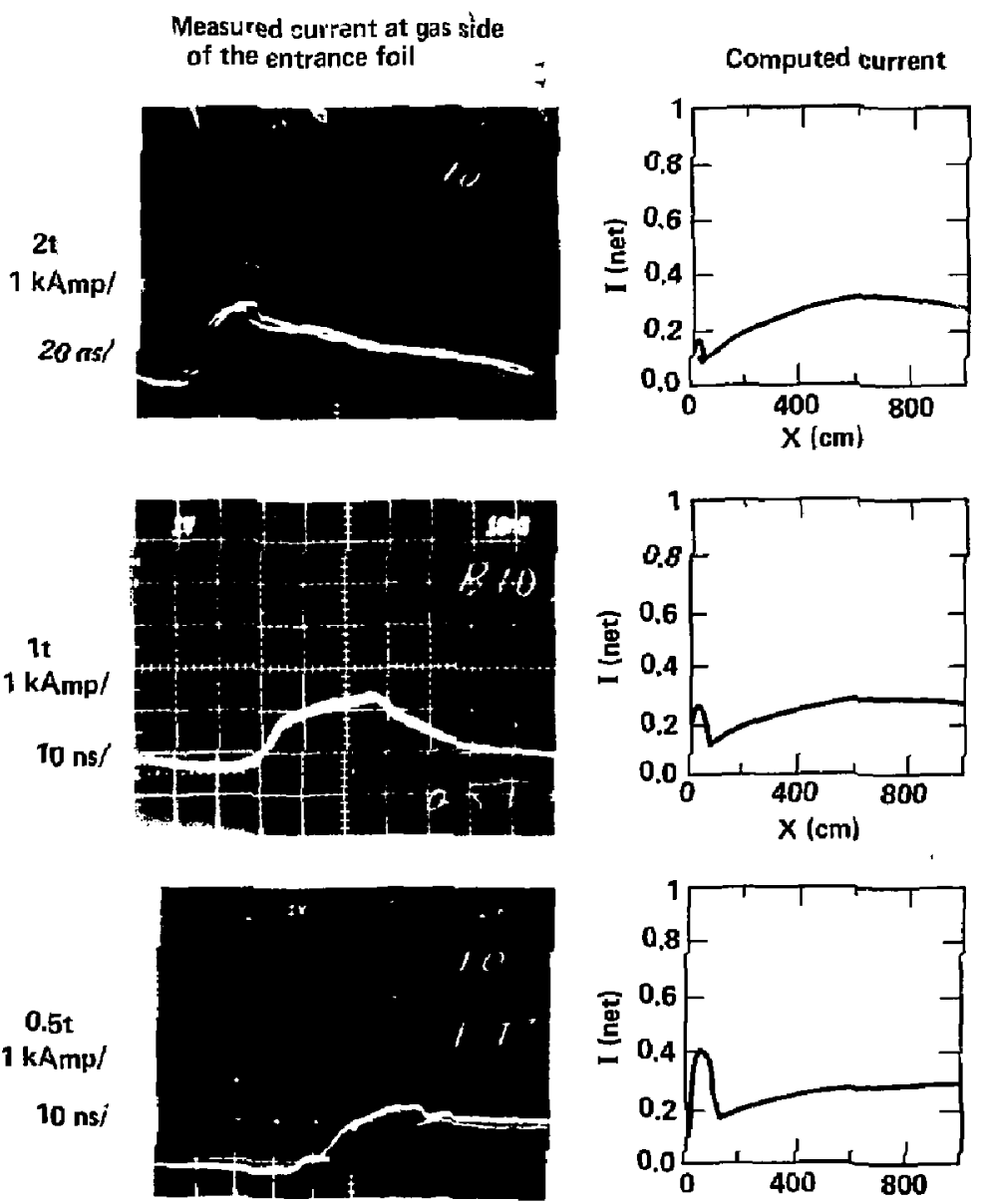

$0.5 \mathrm{t}$

1 kAmp/

$10 \mathrm{nsi}$

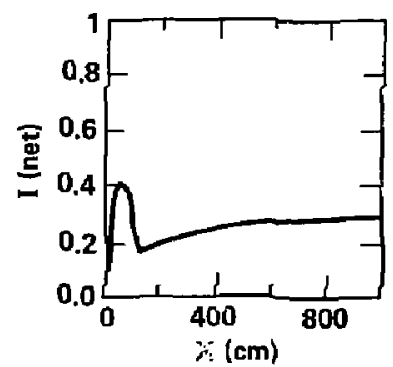

Figure 7C. Comparison of theary with experiment at the naist the beam enters the gas. 

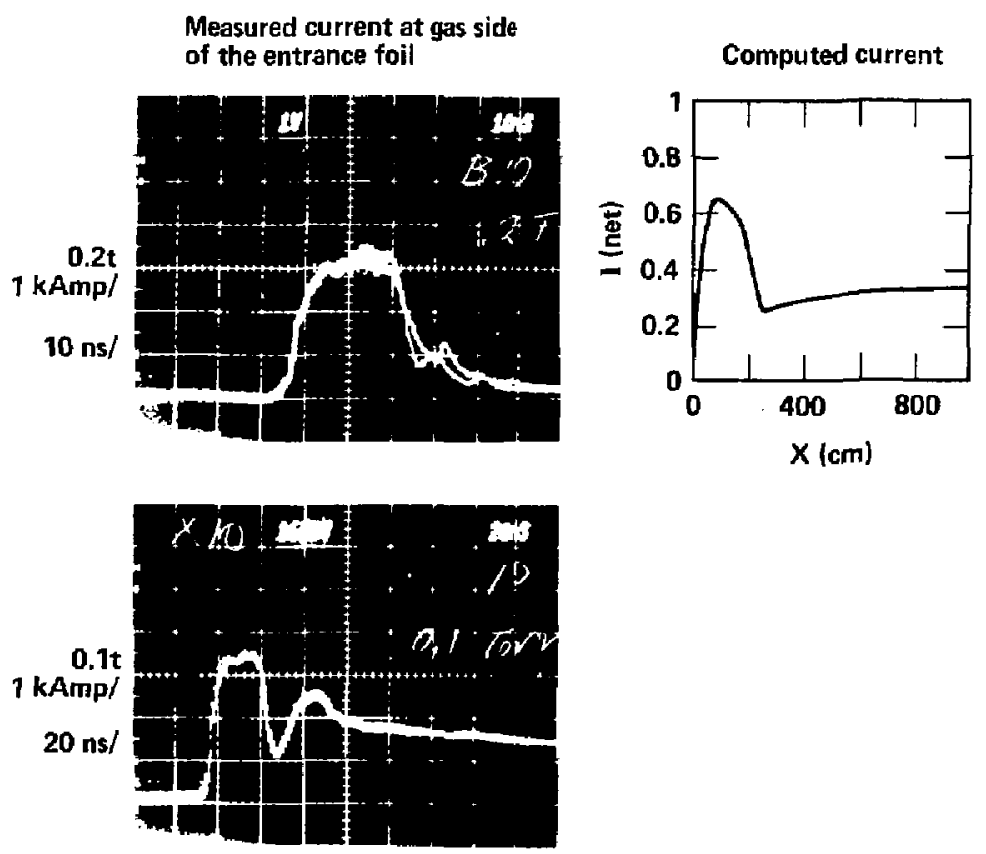

Figure 7D. Comparison of theory with experinent at the point the beam enters the gas. 


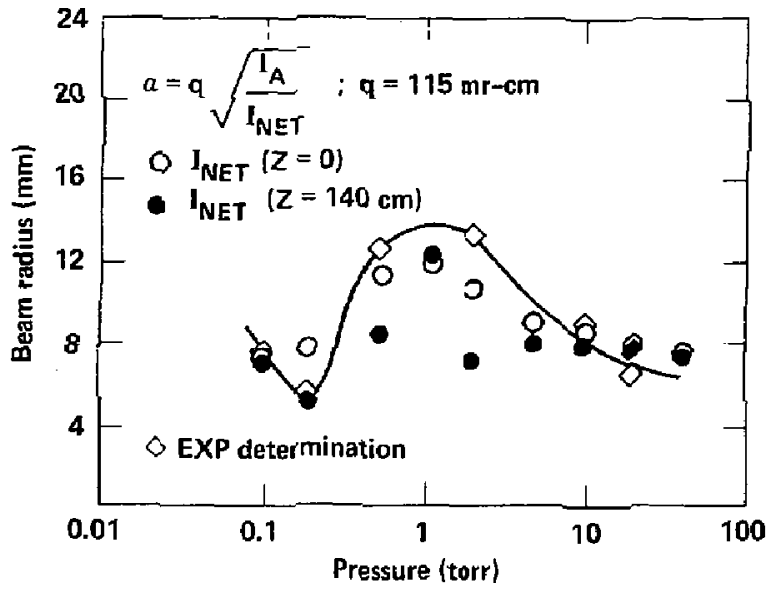

Figure 8. Optically determined beam adius $60 \mathrm{~cm}\left(\sim 1.5 \lambda_{6}\right)$ from entry foil. 